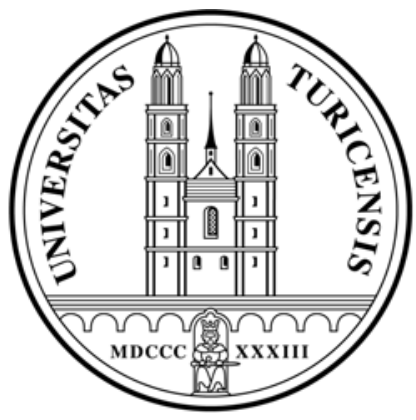

Institute for Empirical Research in Economics

University of Zurich

Working Paper Series

ISSN 1424-0459

Working Paper No. 478

\title{
Reverse Common Ratio Effect
}

Pavlo R. Blavatskyy

February 2010 


\title{
Reverse Common Ratio Effect
}

\author{
Pavlo R. Blavatskyy \\ Institute of Public Finance \\ University of Innsbruck \\ Universitaetsstrasse 15 \\ A-6020 Innsbruck \\ Austria \\ Phone: +43 (0) 5125077156 \\ Fax: +43 (0) 5125072970 \\ e-mail: pavlo.blavatskyy@iew.uzh.ch
}

\begin{abstract}
The results of a new experimental study reveal highly systematic violations of expected utility theory. The pattern of these violations is exactly the opposite of the classical common ratio effect discovered by Allais (1953). Two recent decision theoriesstochastic expected utility theory (Blavatskyy, 2007) and perceived relative argument model (Loomes, 2008)-predicted the existence of a reverse common ratio effect. However, these theories can rationalize only one part of the new experimental data reported in this paper. The other part appears to be neither predicted by existing theories nor documented in the existing empirical studies. (94 words)
\end{abstract}

Keywords: expected utility theory, common ratio effect, Allais paradox, risk, experiment JEL classification codes: C91, D81 


\section{Reverse Common Ratio Effect}

The common ratio effect is a classical example of systematic violations of expected utility theory. In a typical setting, a decision maker has to choose between a sure monetary payoff and a two-outcome lottery that yields a higher outcome with a probability greater than one half (nothing otherwise). The sure monetary payoff is usually selected closely below the expected value of the lottery so that the majority of people choose the sure alternative over the risky lottery. For example, Kahneman and Tversky (1979) report that 80 out of 95 subjects (84.2\%) choose a sure payoff of 3000 Israeli pounds over a risky lottery that yields 4000 Israeli pounds with a probability 0.8 (nothing otherwise).

Now consider a second decision problem, which differs from the first binary choice problem only in one aspect-probabilities of all non-zero outcomes are scaled down by the same common ratio. In the above example from Kahneman and Tversky (1979) probabilities were scaled down by a common factor of 0.25 . In the resulting "scaled-down" problem, subjects had to choose between a safer lottery that yields 3000 Israeli pounds with a probability 0.25 (nothing otherwise) and a riskier lottery that yields 4000 Israeli pounds with a probability 0.2 (nothing otherwise). 65 out of 95 subjects (68.4\%) opted for a riskier lottery.

Expected utility theory implies that people, who opted for a sure monetary payoff (a risky lottery) in the first decision problem, should also choose a safer lottery (a riskier lottery) in the second decision problem. However, many people often choose a sure monetary payoff in the first decision problem and a riskier lottery in the second decision problem. Typically, only few people reveal the opposite tendency to switch from choosing a risky lottery in the "scaled-up" decision problem to choosing a safer lottery in the "scaled-down" decision problem. This asymmetric pattern of expected utility violations is known as the common ratio effect.

The common ratio effect is a seemingly robust experimental finding. Numerous examples of this effect can be found, inter alia, in Ballinger and Wilcox (1997) and Loomes and Sugden (1998). However, two recently proposed decision theories-stochastic 
expected utility theory (Blavatskyy, 2007) and perceived relative argument model (Loomes, 2008)-predict that the common ratio effect disappears if the risky lottery, which is used in the "scaled-up" decision problem, yields a higher outcome with a probability less than one half. Even though the common ratio effect is one of the most frequently researched topics in choice under risk, there are surprisingly few experimental studies that investigate this effect under the above mentioned condition. However, few studies that look at this problem provide tentative evidence in favor of the reverse common ratio effect (e.g. Table 7 in Battalio et al. (1990)).

Given the scarcity of existing experimental evidence, the first objective of this paper is to investigate the common ratio effect when the risky lottery, which is used in the "scaled-up" decision problem, yields a higher outcome with a probability less than one half. We constructed three pairs of common ratio decision problems where the risky lottery yields a higher outcome with a probability 0.25 in the "scaled-up" decision problem. Experimental results clearly showed a strongly asymmetric pattern of the violations of expected utility theory. However, the pattern of these violations was exactly the opposite of the standard common ratio effect. Many subjects switched from choosing a risky lottery in the "scaled-up" decision problem to choosing a safer lottery in the "scaled-down" decision problem. Only few subjects exhibited the opposite switching behavior. Thus, the predictions of two recent decision theories were confirmed-there indeed exists the reverse common ratio effect.

However, this paper also presents new empirical evidence of the reverse common ratio effect, which was neither predicted by any existing decision theory nor documented in existing experimental studies. Consider again the first "scaled-up" decision problem in a classical example of the common ratio effect, where a decision maker chooses between a sure monetary payoff and a risky lottery. The sure monetary payoff is deliberately selected not too far below the expected value of the lottery so that the majority of people are likely to choose the sure alternative over the risky lottery.

In a sense, the common ratio effect is already pre-programmed in this setup. If an overwhelming majority of people choose the sure alternative and virtually no one chooses 
the risky lottery then only two choice patterns are likely to be observed frequently. Those who have chosen the sure alternative in the "scaled-up" problem either choose a safer lottery in the "scaled-down" problem (a pattern consistent with expected utility theory) or switch and choose a riskier lottery in the "scaled-down" problem (a pattern that exhibits the common ratio effect). Since barely anyone chose the risky lottery in the "scaled-up" problem, the reverse common ratio effect is unlikely to be observed.

If this train of thought is correct, we can redesign the classical example to produce a systematic reverse common ratio effect. Suppose that in the first "scaled-up" decision problem a sure monetary payoff is deliberately selected far below the expected value of the risky lottery. In this case, the majority of people are likely to choose the risky lottery over the sure alternative. Now an interesting question: what happens when probabilities of non-zero outcomes are scaled down by the same common ratio?

If the intuition presented above is correct, then a new "scaled-up" decision problem is pre-programmed for the reverse common ratio effect. Since an overwhelming majority of people now choose the risky lottery in the "scaled-up" problem, only two choice patterns are likely to be observed. People who have chosen the risky lottery in the "scaled-up" problem either choose a riskier lottery in the "scaled-down" problem (a pattern consistent with expected utility theory) or switch and choose a safer lottery in the "scaled-down" problem (a pattern that exhibits thereverse common ratio effect). Since few have chosen the sure alternative in the first place, the classical common ratio effect is unlikely to be observed.

Existing non-expected utility theories that account for the classical common ratio effect make a different prediction. Nearly all people who have chosen the risky lottery in the "scaled-up" problem would choose the riskier lottery in the "scaled-down" problem. However, among those few who have opted for the sure alternative in the "scaled-up" problem, many would switch and choose the riskier lottery in the "scaled-down" problem.

We constructed three pairs of common ratio decision problems where a sure monetary payoff is far below the expected value of the risky lottery in the "scaled-up" problem. In all three pairs, we observed highly asymmetric patterns of expected utility 
violations-instances of the reverse common ratio effect greatly outnumbered instances of the classical common ratio effect. This new experimental evidence appears to agree with a simple intuition presented above but it challenges conventional explanations of the common ratio effect.

Encouraged by first experimental results, we also conducted a follow-up experiment to investigate if the same subjects can simultaneously reveal the standard common ratio effect in one type of decision problems and the reverse common ratio effect-in another type of problems. To achieve this objective, we designed the second experiment as follows. In the "scaled-up" problems, subjects choose between the same risky lottery and several monetary payoffs for certain. Sure payoffs are selected so that the maximum (minimum) payoff is far above (below) the expected value of the risky lottery.

When a sure payoff is far above the expected value of the risky lottery in a "scaled-up" problem, nearly all subjects consistently opt for a safer option both in a "scaled-up" and a "scaled-down" problem. However, among those few, who reveal switching behavior, a significant majority manifests the classical common ratio effect. Thus, we successfully replicated results from the existing literature. When a sure payoff is far below the expected value of the risky lottery in a "scaled-up" problem, many subjects opt for a riskier option in both problems. However, subjects, who reveal switching behavior, overwhelmingly display the reverse common ratio effect. Thus, we also replicated results from our first experiment. All in all, the second experiment shows that subjects can exhibit both the standard and the reverse common ratio effect in different decision problems.

The remainder of this paper is organized as follows. The design of our first experimental study is presented in Section 1. The implementation of the first experiment is detailed in Section 2. The results of the first experiment are presented in Section 3. Section 4 discusses experimental results in the context of different decision theories. Section 5 considers implications of this experiment for different models of probabilistic choice. The design of a follow-up experiment is presented in Section 6. Section 7 describes implementation and results of the second experiment. Section 8 concludes. 


\section{Design of the First Experiment}

Six pairs of common ratio decision problems were used in experiment 1 . Pairs 1-3 were designed to test the common ratio effect when a sure monetary payoff is far below the expected value of the risky lottery in the "scaled-up" problem. Pairs 4-6 were designed to test the common ratio effect when the risky lottery yields a higher outcome with a probability less than 0.5 in the "scaled-up" problem. All six pairs of decision problems are presented in Table 1 below.

\begin{tabular}{|l|l|l|l|l|}
\hline \multirow{2}{*}{ Pair \# } & \multicolumn{2}{|c|}{ "Scaled-up" Decision Problem } & \multicolumn{2}{c|}{ "Scaled-down" Decision Problem } \\
\cline { 2 - 5 } & Sure Payoff & Risky Lottery & Safer Lottery & Riskier Lottery \\
\hline 1 & $\$ 60$ & $3 / 4$ chance of $\$ 100$ & $1 / 3$ chance of $\$ 60$ & $1 / 4$ chance of $\$ 100$ \\
2 & $\$ 50$ & $3 / 4$ chance of $\$ 100$ & $1 / 3$ chance of $\$ 50$ & $1 / 4$ chance of $\$ 100$ \\
3 & $\$ 40$ & $3 / 4$ chance of $\$ 100$ & $1 / 3$ chance of $\$ 40$ & $1 / 4$ chance of $\$ 100$ \\
\hline 4 & $\$ 30$ & $1 / 4$ chance of $\$ 100$ & $1 / 3$ chance of $\$ 30$ & $1 / 12$ chance of $\$ 100$ \\
5 & $\$ 20$ & $1 / 4$ chance of $\$ 100$ & $1 / 3$ chance of $\$ 20$ & $1 / 12$ chance of $\$ 100$ \\
6 & $\$ 10$ & $1 / 4$ chance of $\$ 100$ & $1 / 3$ chance of $\$ 10$ & $1 / 12$ chance of $\$ 100$ \\
\hline
\end{tabular}

Table 1 Pairs of decision problems used in the experiment 1

(all lotteries yield \$0 with the remaining probability)

In every pair, the "scaled-down" decision problem is obtained from the "scaledup" problem by dividing the probabilities of all non-zero outcomes by three. Pairs 1-3 have the same risky lottery but a different sure payoff in the "scaled-up" problem. Pairs 4-6 follow a similar design. This is done to rule out the following "indifference" argument.

In principle, an individual who chooses a sure payoff in the "scaled-up" decision problem but switches to choosing a riskier lottery in the "scaled-down" decision problem (or vice versa) does not necessarily violate expected utility theory. This individual may happen to be exactly indifferent between the sure payoff and a risky lottery in the "scaledup" problem. Under expected utility theory, she is then also indifferent between both alternatives in the "scaled-down" decision problem. Since an individual who is exactly indifferent may choose in any way she likes, a revealed switching behavior can, in fact, be consistent with expected utility theory. 
This does not strike us as a very plausible explanation (many people happen to be exactly indifferent and, when indifferent, they randomize their choices with different probabilities in different decision problems). Even though this appears to be an unlikely scenario, we designed our experiment to control for this explanation because none of the existing experimental studies have done so.

To rule out the "indifference" argument, we used the same risky lottery but several different sure payoffs in the "scaled-up" problem. Thus, if a revealed switching behavior is a mere reflection of indifference, it can be observed, at most, in one pair of decision problems. If it is observed in two or three pairs, it cannot be attributed to indifference because an individual cannot be simultaneously indifferent between a risky lottery and two or three distinct monetary amounts.

Since not all subjects may be familiar or comfortable with probability calculus, the probability information was conveyed through a composition of red and black cards. Figure 1 shows a "scaled-down" problem from pair 1 as it was displayed in the experiment.

\section{Question 2}

Please choose your preferred alternative:

Left Alternative

An experimenter shows you 3 cards.

One card is red and 2 cards are black.

All 3 cards are faced down and reshuffled.

Then you draw one card. You receive $\$ 60$ if it is red and you receive nothing if it is black.

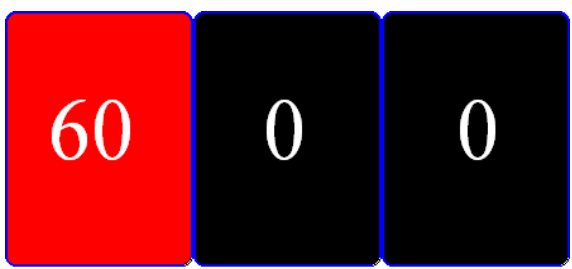

I choose: $\square$ Left Alternative

\section{Right Alternative}

An experimenter shows you 4 cards.

One card is red and 3 cards are black.

All 4 cards are faced down and reshuffled.

Then you draw one card. You receive $\$ 100$ if it is red and you receive nothing if it is black.

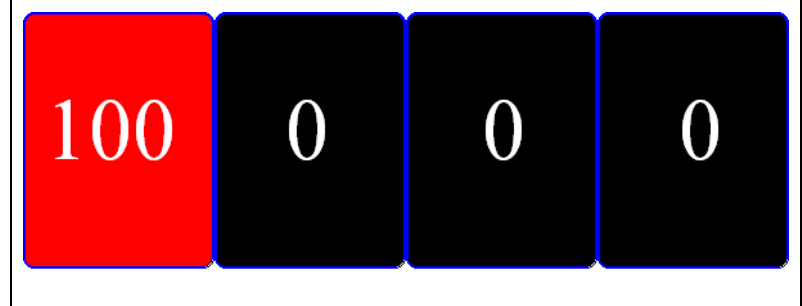

Right Alternative

Figure $1 \mathrm{~A}$ sample question as displayed in the experiment 


\section{Implementation of the First Experiment}

The experiment 1 was conducted in the experimental laboratory of the University of Zurich. Altogether, 70 undergraduate students took part in two experimental sessions, which were conducted at the same afternoon. 31 out of 70 subjects (44.3\%) were female. The average age of experimental participants was 21.8 years (minimum age was 18 years and maximum age was 38 years). 4 out of 70 subjects (5.7\%) were economics majors. The majority of subjects had some previous experience with economic experiments but none of them had participated in an experiment on choice under risk.

At the beginning of each session, experimental instructions were read aloud (text of instructions is presented in the Appendix). Then, 12 binary choice problems (from Table 1) were presented to subjects in a random order on a computer screen. The appearance of safer or riskier lotteries in the left or the right part of the screen was randomized.

After answering all binary choice questions and filling out a short sociodemographic questionnaire, each subject spun a roulette wheel. The number of sectors on the roulette wheel corresponded to the total number of questions asked in the experiment. The question, which was randomly selected on the roulette wheel, was played out for real money.

Subjects who opted for a sure monetary payoff in the selected question received this amount in cash. Subjects who opted for a lottery in the selected question were shown a composition of red and black cards (from a deck of standard playing cards). These cards were then reshuffled and subjects had to draw one card. Depending on the color of their drawn card, they received the corresponding payoff. Upon observing the color of their drawn card, subjects inspected the color of all remaining cards to verify that the composition of red and black cards did not change after reshuffling.

Each experimental session lasted about one hour. However, more than half of this time was spent on using physical randomization devices at the end of the experiment. One experimental dollar was exchanged for $1 \mathrm{CHF}$ (approximately 0.85 USD). On average, subjects earned 52.14 CHF (including a $10 \mathrm{CHF}$ show-up fee). A median payoff was $50 \mathrm{CHF}$. A maximum payoff was $110 \mathrm{CHF}$ and a minimum payoff was $10 \mathrm{CHF}$. 


\section{Results of the First Experiment}

In every pair of decision problems, subjects can reveal one of four possible choice patterns. First, subjects can choose a sure payoff in the "scaled-up" problem and a safer lottery in the "scaled-down" problem. We label this pattern as $\mathbf{S}$ for "safe". Second, subjects can choose a sure payoff in the "scaled-up" problem and a riskier lottery in the "scaled-down" problem. We label this pattern as CR for "common ratio". Third, subjects can choose a risky lottery in the "scaled-up" problem and a safer lottery in the "scaleddown" problem. We label this pattern as RCR for "reverse common ratio". Fourth, subjects can choose a risky lottery in the "scaled-up" problem and a riskier lottery in the "scaled-down" problem. We label this pattern as $\mathbf{R}$ for "risky". Table 2 shows the observed frequency of these choice patterns in pairs 1-6.

\begin{tabular}{|c|c|c|c|c|c|c|}
\hline \multirow{2}{*}{ Pair \# } & \multicolumn{4}{|c|}{ Choice Pattern } & \multirow{2}{*}{ Conlisk $z$} & \multirow{2}{*}{$p$-value } \\
\hline & $S$ & CR & RCR & $\mathbf{R}$ & & \\
\hline 1 & 24 & 2 & 23 & 21 & -4.8214 & $7.13 \mathrm{E}-07$ \\
\hline 2 & 15 & 4 & 21 & 30 & -3.6944 & 1.10E-04 \\
\hline 3 & 8 & 4 & 24 & 34 & -4.2062 & $1.30 \mathrm{E}-05$ \\
\hline 4 & 65 & 1 & 3 & 1 & -1 & 0.1587 \\
\hline 5 & 49 & 2 & 12 & 7 & -2.8002 & 0.0026 \\
\hline 6 & 18 & 5 & 18 & 29 & -2.8447 & 0.0022 \\
\hline
\end{tabular}

Table 2 Observed frequency of choice patterns in experiment 1

Note that the frequency of the $\mathbf{S}$ pattern decreases and the frequency of the pattern $\mathbf{R}$ increases from pair 1 to pair 3 (and from pair 4 to pair 6 ). In other words, subjects respond well to the incentives. When a safer alternative is getting progressively worse, subjects choose a riskier alternative more frequently.

In every pair of decision problems, the RCR pattern is observed more often than the CR pattern. In other words, we observe systematic violations of expected utility theory with patterns opposite of the classical common ratio effect. For every pair of decision problems, Table 2 also shows Conlisk $z$-statistic (Conlisk, 1989) and its $p$-value. For 5 out of 6 pairs there is a highly significant difference between RCR and $\mathbf{C R}$ frequencies. 
In fact, the observed reverse common ratio effect is so strong that modal choice changes from a "scaled-up" to a "scaled-down" problem in three pairs of decision problems. In pair 1 , only 26 out of 70 subjects (37.1\%) opt for a sure payoff in the "scaledup" problem but 47 out of 70 subjects (67.1\%) choose a safer lottery in the "scaled-down" problem. In pair 2, 19 out of 70 subjects (27.1\%) opt for a sure payoff in the "scaled-up" problem but 36 out of 70 subjects (51.4\%) choose a safer lottery in the "scaled-down" problem. Finally, in pair 6, 23 out of 70 subjects (32.9\%) opt for a sure payoff in the "scaled-up" problem but 36 out of 70 subjects (51.4\%) choose a safer lottery in the "scaled-down" problem. Our first result can be summarized as follows.

\section{Result 1 Reverse common ratio effect occurs significantly more often than the standard common ratio effect.}

Individual choice patterns ("raw" experimental data) are presented in Table 5 and Table 6 in the Appendix. 15 out of 70 subjects (21.4\%) made consistent choices in all six pairs of decision problems. Their choice patterns are presented in Table 6. Among these subjects, 5 subjects consistently choose a safer alternative in every binary choice question. This was a modal individual choice pattern.

The remaining 55 subjects (78.6\%) revealed a switching choice pattern (CR or $\mathbf{R C R}$ ) in at least one pair of decision problems. Subjects' choice patterns of this kind are presented in Table 5 . In particular, 17 subjects (24.3\%) revealed a switching behavior that may be reconciled with expected utility theory under the assumption that these subjects are exactly indifferent in one of common ratio pairs 1-3 and/or in one of common ratio pairs 4-6. The switching behavior of the remaining 38 subjects (54.3\%) cannot be rationalized by expected utility theory even if we assume the possibility of exact indifferences. Thus, we can summarize our second result, which has not yet been established in the existing experimental studies on the Allais paradox.

Result 2 Most systematic violations of expected utility theory cannot be attributed to pure indifferences. 
Only 7 out of 70 subjects (10.0\%) revealed the CR pattern in at least one pair of decision problems but did not reveal the RCR pattern in any of the pairs. Another 8 subjects (11.4\%) revealed both CR and RCR patterns in different pairs of decision problems. Finally, 40 out of 70 subjects (57.1\%) revealed only RCR patterns in one or several pairs of decision problems. Thus, Result 1 holds both on individual and on the aggregate level.

So far, we looked only at the consistency of individual responses within pairs of common ratio problems. However, we can also look at consistency in "scaled-up" problems as well as in "scaled-down" problems across pairs 1-3 and across pairs 4-6. The essence of this check is as follows; if an individual chooses a $3 / 4$ chance of $\$ 100$ over $\$ 60$ for sure, and she has rational preferences of some kind, then she should choose a $3 / 4$ chance of $\$ 100$ over $\$ 50$ (or $\$ 40$ ) for sure. A similar consistency may be expected in the "scaleddown" problems. If an individual chooses a $1 / 4$ chance of $\$ 100$ over a $1 / 3$ chance of $\$ 60$ then she should also choose a $1 / 4$ chance of $\$ 100$ over a $1 / 3$ chance of $\$ 50$ (or $\$ 40)$.

Overall, 15 out of 70 subjects (21.4\%) made inconsistent choices across pairs of decision problems. This aligns with other experimental studies. Ballinger and Wilcox (1997) report a median switching rate of $20.8 \%$. Loomes and Sugden (1998) report an average inconsistency rate of $18.3 \%$. Most cross-pair inconsistent choices occurred in "scaled-down" problems from pairs 1-3. There are only 3 cross-pair inconsistent choices in pairs 4-6 and there are 4 cross-pair inconsistent choices in "scaled-up" problems from pairs 1-3.

Notice that an inconsistency rate across pairs ( $21.4 \%$ of subjects) is well below the switching rate within pairs (78.6\% of subjects). This observation tentatively suggests that the reverse common ratio effect is embedded in individual preferences and it is not caused by random errors or noise. However, it is more appropriate to postpone this discussion till Section 5. Meanwhile, let us simply summarize our third result as follows. Result 3 An inconsistency rate across pairs is much lower compared to the switching rate within pairs. 


\section{Implications for Decision Theories}

Classical Allais paradox is the ultimate raison d'être for non-expected utility theories (e.g. Kahneman and Tversky, 1979; Gul, 1991). Hence, one cannot expect much predictive power from these theories as far as the reverse common ratio effect is concerned. In fact, it turns out that standard models of decision making under risk cannot account for any choice patterns observed in pairs 1-6.

Most standard decision theories postulate that the utility of lottery $L$ that yields a $p$ chance of $\$ x$ ( $\$ 0$ otherwise) is given by the following simple formula:

$$
U(L)=w(p) \cdot u(x)+(1-w(p)) \cdot u(0),
$$

where $w:[0,1] \rightarrow \mathbb{R}$ and $u: \mathbb{R} \rightarrow \mathbb{R}$ are two functions that differ across decision theories.

For example, under expected utility theory $w(p)=p$ for all $p \in[0,1]$. Rankdependent utility theory (Quiggin, 1981) and cumulative prospect theory (Tversky and Kahneman, 1992) assume that function $w($.$) is sub-additive, i.e. w(p)+w(q) \geq w(p+q)$ for any $p, q \in[0,1]$ and $p+q \leq 1$. Yaari (1987) assumes that $u(x)=x$. In disappointment aversion theory (Gul, 1991) function $w($.$) takes a specific form:$

$$
w(p)=\frac{p}{1+(1-p) \cdot \beta},
$$

with $\beta>-1$. Prospective reference theory (Viscusi, 1989) assumes that function $w($.$) takes$ another specific form: $w(0)=0, w(1)=1$ and $w(p)=\lambda p+(1-\lambda) / 2$ for all $p \in(0,1)$, where $\lambda \in[0,1]$.

If utility of a two-outcome lottery is given by formula (1), an individual chooses a risky lottery over a sure payoff in the "scaled-up" problem from pair $i \in\{1,2,3\}$ only if

$$
w\left(\frac{3}{4}\right) \geq \frac{u(70-10 i)-u(0)}{u(100)-u(0)} .
$$

Similarly, an individual chooses a safer lottery over a riskier lottery in the "scaled-down" problem from pair $i \in\{1,2,3\}$ only if

$$
\frac{u(70-10 i)-u(0)}{u(100)-u(0)} \geq \frac{w(1 / 4)}{w(1 / 3)} .
$$


Thus, $\mathbf{R C R}$ pattern may be observed in pair $i \in\{1,2,3\}$ only if the following condition holds:

$$
w\left(\frac{3}{4}\right) \geq \frac{u(70-10 i)-u(0)}{u(100)-u(0)} \geq \frac{w(1 / 4)}{w(1 / 3)} .
$$

Under expected utility theory, function $w($.$) is linear and the left hand side of (5)$ is exactly equal to the right hand side of (5), that is to three quarters. In this case, condition (5) can be satisfied only if the utility ratio in the middle of (5) equals three quarters. Thus, condition (5) may hold for one number $i \in\{1,2,3\}$ but it cannot hold for another number $j \in\{1,2,3\}, j \neq i$. In other words, expected utility theory cannot account for the RCR pattern that is observed in two or three pairs of decision problems. Yet, the majority of individual choice patterns from our experiment have exactly this property (Result 2 from the previous section).

Under disappointment aversion theory, function $w($.$) is given by (2). In this case,$ the left hand side of (5) is greater than or equal to the right hand side of (5) if and only if either $\beta=0$ (that is when disappointment aversion theory coincides with expected utility theory) or $\beta \leq-1$, which is inadmissible parameterization. Thus, disappointment aversion theory does not offer any improvement over expected utility theory when it comes to explaining the reverse common ratio effect in pairs 1-3.

The same conclusion holds for prospective reference theory. In this case, the left hand side of (5) is greater than or equal to the right hand side of (5) if and only if either $\lambda=1$ (i.e. when prospective reference theory coincides with expected utility theory) or $\lambda \geq 6$, which is an inadmissible parameterization.

Under rank-dependent utility theory and cumulative prospect theory with socalled Quiggin probability weighting function $w(p)=p^{\gamma} /\left(p^{\gamma}+(1-p)^{\gamma}\right)^{1 / \gamma}, p \in[0,1]$, $\gamma \leq 1$, the left hand side of (5) is greater than or equal to the right hand side of (5) if and only if $\gamma \geq 1$. Thus, $\gamma=1$ is the only admissible parameter value under which condition (5) may hold. Again, this is a special case that corresponds to expected utility theory.

More generally, for any sub-additive probability weighting function $w($.$) that$ satisfies $w(1 / 3)=1 / 3$, we have $w(1 / 4) / w(1 / 3) \geq w(3 / 4)$. However, condition (5) requires 
that this weak inequality holds with a reverse sign. Hence, condition (5) may hold only in one case when $w(1 / 4) / w(1 / 3)=w(3 / 4)$. In this case, rank-dependent utility theory and cumulative prospect theory can rationalize the $\mathbf{R C R}$ pattern in one pair $i \in\{1,2,3\}$ but they cannot account for when the RCR pattern is observed in two or three pairs of decision problems. In other words, both of these theories run into the same difficulties as expected utility theory does.

Standard models of decision making under risk also have difficulties with choice patterns revealed in pairs 4-6. If utility of a two-outcome lottery is given by formula (1), then the reverse common ratio effect is observed in pair $i \in\{4,5,6\}$ only if the following condition holds:

$$
w\left(\frac{1}{4}\right) \geq \frac{u(70-10 i)-u(0)}{u(100)-u(0)} \geq \frac{w(1 / 12)}{w(1 / 3)}
$$

Given an apparent similarity between (6) and (5), it is immediately clear that all decision theories considered so far can satisfy condition (6), at most, only for one pair $i \in\{4,5,6\}$. Thus, these theories cannot rationalize the reverse common ratio effect observed in two or three pairs 4-6.

However, there are two recent decision theories that predicted the existence of the reverse common ratio effect in decision problems similar to our pairs 4-6. One of them is a perceived relative argument model developed by Loomes (2008). According to this model, an individual chooses a risky lottery over a sure payoff in the "scaled-up" problem from pair $i \in\{4,5,6\}$ only if the following condition holds:

$$
\xi(30+10 i, 70-30 i) \geq 3
$$

where $\xi: \mathbb{R} \times \mathbb{R} \rightarrow \mathbb{R}$ is a function that captures payoff perceptions. The same individual chooses a safer lottery over a riskier lottery in the "scaled-down" problem from pair $i \in\{4,5,6\}$ only if

$$
3^{1 / 3^{\alpha}} \geq \xi(30+10 i, 70-30 i)
$$

where $\alpha \leq 0$ is a parameter that captures probability perceptions. 
Combining inequalities (7) and (8) we obtain a necessary condition for observing the reverse common ratio effect in pair $i \in\{4,5,6\}$ :

$$
3^{1 / 3^{\alpha}} \geq \xi(30+10 i, 70-30 i) \geq 3 .
$$

If $\alpha<0$, the left hand side of (9) is always greater than the right hand side of (9). Thus, if value $\xi(30+10 i, 70-30 i)$ happens to be in the interval $\left[3,3^{1 / 3^{\alpha}}\right]$, the reverse common ratio effect occurs in pair $i \in\{4,5,6\}$. Clearly, this can also happen for another number $j \in\{4,5,6\}, j \neq i$. Thus, a perceived relative argument model can account for the $\mathbf{R C R}$ pattern that is observed in two or three pairs 4-6.

However, this model cannot rationalize the reverse common ratio effect in pairs 13. According to this model, a necessary condition for the RCR pattern in pair $i \in\{1,2,3\}$ is

$$
\left(\frac{1}{3}\right)^{\frac{1}{3^{\alpha}}} \geq \xi(30+10 i, 70-30 i) \geq \frac{1}{3} .
$$

For any $\alpha<0$, the left hand side of (10) is less than one third, i.e. condition (10) cannot hold. If $\alpha=0$, then the left hand side of (10) equals one third, and condition (10) may be satisfied for one number $i \in\{1,2,3\}$. This is a special case when the model coincides with expected utility theory. In sum, a perceived relative argument model can account for the reverse common ratio effect in pairs 4-6 but does not offer a comparative advantage over expected utility theory when it comes to choice patterns in pairs 1-3.

Overall, the results of this experimental study have quite negative (though perhaps unsurprising) implications for the existing decision theories. Most non-expected utility theories were designed to account for the Allais paradox. As a consequence, they do not provide any improvement over expected utility theory when it comes to rationalizing the reverse common ratio effect. Only one theory-perceived relative argument modelwas partially successful in explaining new experimental data.

Given that there appears no promising decision theoretical account of the data, it is natural to consider other possible explanations. Namely, we shall investigate whether the reverse common ratio effect could be an artifact of random errors, noise, imprecise or random preferences. Since such theories require quite a different modeling approach, their analysis is separate, located in the next section of the paper. 


\section{Implications for Probabilistic Models}

Revealed choice patterns that appear at the first sight as systematic violations of expected utility theory can, in fact, be a byproduct of random errors, noise, imprecise or random preferences. In particular, Loomes (2005) shows that a strong utility model can generate certain types of the classical common ratio effect. It turns out that this model can also account for the reverse common ratio effect.

For example, consider the "scaled-up" problem from pair $i \in\{1, \ldots, 6\}$. In a strong utility model, an individual chooses a risky lottery over a sure payoff with a probability $F\left(\Delta E U_{i}\right)$, where $F: \mathbb{R} \rightarrow[0,1]$ is a non-decreasing function (typically interpreted as a distribution function of random errors) and $\Delta E U_{i}$ denotes the difference in expected utility between a risky lottery and a sure payoff in the "scaled-up" problem from pair $i \in\{1, \ldots, 6\}$. In the "scaled-down" problem, this individual chooses a riskier lottery over a safer lottery with a probability $F\left(\Delta E U_{i} / 3\right)$. Thus, the RCR pattern is observed in pair $i \in\{1, \ldots, 6\}$ with a probability

$$
\operatorname{Pr}\{\mathbf{R C R}\}=F\left(\Delta E U_{i}\right) \cdot\left[1-F\left(\Delta E U_{i} / 3\right)\right]
$$

and the CR pattern is observed in pair $i \in\{1, \ldots, 6\}$ with a probability

$$
\operatorname{Pr}\{\mathbf{C R}\}=\left[1-F\left(\Delta E U_{i}\right)\right] \cdot F\left(\Delta E U_{i} / 3\right) .
$$

In principle, two cases are possible. First, the difference $\Delta E U_{i}$ may be negative. In this case, $F\left(\Delta E U_{i}\right) \leq F\left(\Delta E U_{i} / 3\right)$ and formulas (11) and (12) imply that $\operatorname{Pr}\{\mathbf{C R}\} \geq \operatorname{Pr}\{\mathbf{R C R}\}$. This is the case discussed in Loomes (2005). In the "scaled-up" problem, an individual finds the sure option to be more attractive in terms of expected utility. So the individual chooses the sure payoff, except for an occasional random error. When the probabilities of all non-zero outcomes are scaled down by the same common ratio, the difference in expected utility (favoring the safer option) is diluted and more random errors are likely to occur. These random errors generate a higher frequency of $\mathbf{C R}$ patterns (compared to the frequency of RCR patterns). An experimenter, who observes only revealed choice patterns, detects what appears to be a standard common ratio effect. 
On the other hand, there may be a second possibility. Suppose that an experimenter deliberately picks a sure monetary payoff far below the expected value of a lottery in the "scaled-up" decision problem (as in pairs 1-3). In this case, the difference $\Delta E U_{i}$ is likely to be positive. Since function $F($.$) is non-decreasing, this implies that$ $F\left(\Delta E U_{i}\right) \geq F\left(\Delta E U_{i} / 3\right)$ and $\operatorname{Pr}\{\mathbf{R C R}\} \geq \operatorname{Pr}\{\mathbf{C R}\}$ due to formulas (11) and (12). Hence, the $\mathbf{R C R}$ pattern occurs more frequently compared to the $\mathbf{C R}$ pattern.

This case formally captures a simple intuition behind our experimental design that was described in the introduction. If a sure payoff in the "scaled-up" problem is unattractive compared to a risky lottery, a decision maker opts for the risky alternative almost all the time (except for an occasional random error). This behavior changes when the probabilities of all non-zero outcomes are scaled down by the same common ratio. The difference in expected utility, which favors the riskier option, is now diminished. More random errors are likely to occur. In the extreme case, the probability of choosing a safer alternative can increase up to 0.5 . Thus, random errors generate a high incidence of $\mathbf{R C R}$ patterns and a low incidence of CR patterns. In the experimental data this appears as a systematic reverse common ratio effect.

Even though a strong utility model can account for Result 1, it faces a serious challenge when it comes to Result 3. For example, consider an individual who chooses a $3 / 4$ chance of $\$ 100$ over $\$ 60$ for sure (in the "scaled-up" problem from pair 1 ) but prefers $\$ 50$ for sure over a $3 / 4$ chance of $\$ 100$ (in the "scaled-up" problem from pair 2). According to a strong utility model, the likelihood of such cross-pair inconsistency happening is given by

$$
F\left(\Delta E U_{1}\right) \cdot\left[1-F\left(\Delta E U_{2}\right)\right] \text {. }
$$

Table 2 shows that 44 out of 70 subjects (62.9\%) opted for a risky lottery in the "scaled-up" problem from pair 1 and 19 out of 70 subjects (27.1\%) opted for a sure payoff in the "scaled-up" problem from pair 2. According to formula (12), we can expect around 12 cross-pair inconsistencies in this case. However, Table 5 shows that there were only 2 such cross-pair inconsistencies. Fisher's exact test $p=0.003$ shows that the frequency of actual cross-pair inconsistencies is significantly below the model expectation. 
A similar picture emerges if we consider inconsistency rates across other pairs of decision problems. A strong utility model always predicts too many cross-pair inconsistencies. Loomes and Sugden (1998) reach a similar conclusion, though with a completely different experimental setup.

To sum up, a strong utility model can generate a systematic reverse common ratio effect. However, this model also predicts too many cross-pair inconsistencies. Hence, the reverse common ratio effect does not appear to result from random errors or noise (at least not when those are modeled through strong utility).

A strong utility model can explain why RCR patterns occur more frequently than CR patterns but it cannot account for a very asymmetric reverse common ratio effect when the majority of people choose a risky lottery in the "scaled-up" problem and, at the same time, the majority of people opt for a safer lottery in the "scaled-down" problem. For example, such a strong reverse common ratio effect is observed in pair 1 (cf. Table 2). To see why the model fails to account for such patterns, consider an example when the difference $\Delta E U_{i}$ is positive for some $i \in\{1, \ldots, 6\}$. We already established that in this case a strong utility model predicts that RCR patterns occur more frequently than CR patterns. In the "scaled-down" problem, a riskier lottery is chosen with a probability $F\left(\Delta E U_{i} / 3\right) \geq F(0)=1 / 2$. Hence, even though RCR patterns occur more frequently, choosing a riskier lottery should be a modal choice pattern in the "scaled-down" problem. Blavatskyy (2007) modifies a strong utility model to get rid of this restriction. His modification-a stochastic expected utility theory-also explicitly predicts that a highly asymmetric classical common ratio effect (with a switching modal choice pattern) disappears if the risky lottery, which is used in the "scaled-up" decision problem, yields a higher outcome with a probability less than one half (Blavatskyy, 2007, p. 267). However, a stochastic expected utility theory does not offer any comparative advantage over a strong utility model when it comes to explaining Result 3. Like its parent model, a stochastic expected utility theory cannot explain why so many people reveal inconsistent choice patterns within but not across pairs of decision problems. 
A model of random preferences that admit expected utility representation (e.g. Loomes and Sugden, 1995; Gul and Pesendorfer, 2006) predicts equal frequencies of CR and RCR patterns so it cannot even account for Result 1. Consider an individual who chooses a lottery over a sure amount in the "scaled-up" problem. Under a random preference model, the likelihood of such a choice is driven by the likelihood of selecting a preference relation such that the lottery is preferred over the sure amount in the "scaledup" problem. However, any such preference relation is also one where a riskier lottery is preferred over a safer lottery in the "scaled-down" problem, provided that all preference relations are of expected utility type. Hence, the chance of choosing a riskier option is the same in the "scaled-up" and "scaled-down" problem and there cannot be any asymmetry in CR and RCR patterns (see also Loomes (2005)).

MacCrimmon and Smith (1986) put forward a model of imprecise preferences that was recently popularized in Butler and Loomes (2007). This model is illustrated on Figure 2.

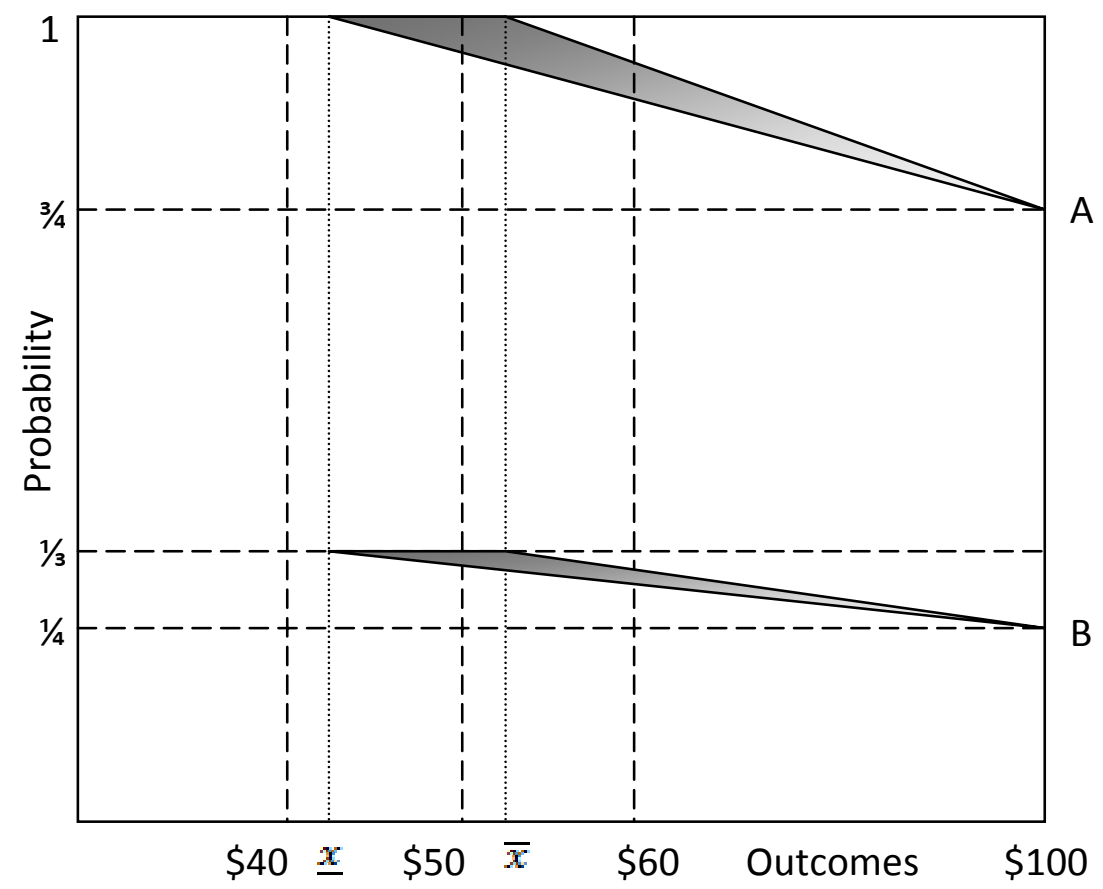

Figure 2 Model of imprecise preferences 
The vertical axis on Figure 2 shows probability values. The horizontal axis on Figure 2 shows outcomes. For example, point A on Figure 2 represents a $3 / 4$ chance of $\$ 100$ (\$0 otherwise). In a standard microeconomic model (with complete, transitive and continuous preferences) there is a unique indifference curve that connects point $A$ to some point on the horizontal line "1". This point represents a (unique) certainty equivalent of a lottery that offers a $3 / 4$ chance of $\$ 100$. In a model with imprecise preferences, there is no unique certainty equivalent of a lottery. Rather, there is a range of possible certainty equivalents that are represented as an interval $[\underline{x}, \bar{x}]$ on Figure 2 .

For example, an individual with imprecise preferences, depicted on Figure 2, always chooses $\$ 60$ over a $3 / 4$ chance of $\$ 100$. Also, she always chooses a $3 / 4$ chance of $\$ 100$ over $\$ 40$. However, when it comes to choosing between a $3 / 4$ chance of $\$ 100$ and $\$ 50$ for sure, she sometimes opts for the risky lottery and sometimes for the sure payoff. So, this individual never exhibits cross-pair inconsistencies. Of course, if the width of an imprecision interval $[\underline{x}, \bar{x}]$ is sufficiently large so that it contains, for example, both $\$ 60$ and $\$ 50$, an individual can reveal inconsistent choices across pairs 1 and 2 . Yet, it is clear that the model of imprecise preferences has some potential for explaining Result 3 when few people have wide imprecision intervals.

Can the model also account for Result 1? Point B on Figure 2 represents a $1 / 4$ chance of $\$ 100$ (\$0 otherwise). Again, point B is connected to some imprecision interval on the horizontal line " $1 / 3$ ". If this imprecision interval coincides with an interval $[\underline{x}, \bar{x}]$, as depicted on Figure 2, there is no systematic common ratio effect in pairs 1-3. Otherwise, either CR patterns occur more frequently than RCR patterns or vice versa.

Unfortunately, neither MacCrimmon and Smith (1986) nor Butler and Loomes (2007) make any specific prediction about the location of an imprecision interval for point $B$ in relation to that for point $A$. In other words, a model of imprecise preferences can (potentially) account for the reverse common ratio effect but it does not predict such an effect. Nonetheless, among all probabilistic models, a model of imprecise preferences may be our best shot since it is the only model that can reconcile Results 1 and 3 together. 


\section{Design of the Second Experiment}

The standard common ratio effect is often found in decision problems similar to the example from Kahneman and Tversky (1979) presented in the introduction. We also discovered the reverse common ratio effect in decision problems presented in Table 1. Is it possible that the same subjects exhibit two opposite common ratio effects in different decision problems? We investigate this issue in our second experiment.

Five pairs of common ratio problems were used in experiment 2. Pairs 7 and 8 were designed so that a sure payoff is above or equal to the expected value of the risky lottery in the "scaled-up" problem. Common ratio problems of this type were extensively studied in the existing literature. Typically, subjects reveal the classical common ratio effect is such problems, e.g. Loomes and Sugden (1998).

Pairs 9-11 were designed so that a sure payoff is far below the expected value of the risky lottery in the "scaled-up" problem. Pairs 9-11 are similar to pairs 1-3 in experiment 1 . In fact, we obtained pairs 9-11 by scaling down all payoffs in pairs 1-3 by a factor of 2.5 and rounding all payoffs to multiples of $\$ 5$. For reasons already explained above, we expect that subjects reveal the reverse common ratio effect in pairs 9-11.

All five pairs of decision problems are presented in Table 3 below. Note that all five pairs 7-11 have the same risky lottery but different sure payoffs in the "scaled-up" problem. Thus, even if subjects reveal switching behavior as a consequence of exact indifference, they can do it, at most, only in one pair of common ratio problems. Exactly as in experiment 1 , we conveyed probability information to subjects by means of a composition of red and black cards (cf. Figure 1).

\begin{tabular}{|l|l|l|l|l|}
\hline \multirow{2}{*}{ Pair \# } & \multicolumn{2}{|c|}{ “Scaled-up" Decision Problem } & \multicolumn{2}{c|}{ "Scaled-down" Decision Problem } \\
\cline { 2 - 5 } & Sure Payoff & Risky Lottery & Safer Lottery & Riskier Lottery \\
\hline 7 & $\$ 35$ & $3 / 4$ chance of $\$ 40$ & $1 / 3$ chance of $\$ 35$ & $1 / 4$ chance of $\$ 40$ \\
8 & $\$ 30$ & $3 / 4$ chance of $\$ 40$ & $1 / 3$ chance of $\$ 30$ & $1 / 4$ chance of $\$ 40$ \\
9 & $\$ 25$ & $3 / 4$ chance of $\$ 40$ & $1 / 3$ chance of $\$ 25$ & $1 / 4$ chance of $\$ 40$ \\
10 & $\$ 20$ & $3 / 4$ chance of $\$ 40$ & $1 / 3$ chance of $\$ 20$ & $1 / 4$ chance of $\$ 40$ \\
11 & $\$ 15$ & $3 / 4$ chance of $\$ 40$ & $1 / 3$ chance of $\$ 15$ & $1 / 4$ chance of $\$ 40$ \\
\hline
\end{tabular}

Table 3 Pairs of decision problems used in the experiment 2 (all lotteries yield \$0 with the remaining probability) 


\section{Implementation and Results of the Second Experiment}

Experiment 2 was conducted in Behavioral Research Laboratory at the Columbia Business School (Columbia University in New York). ${ }^{1}$ Altogether, 93 students took part in five experimental sessions, which were conducted on three consecutive days. 57 out of 93 subjects (61.3\%) were female. The average age of experimental participants was 23.3 years (minimum age was 18 years and maximum age was 47 years). 8 out of 93 subjects (8.6\%) studied economics or business administration.

Experiment 2 was conducted with the same instructions, computerized procedure and physical randomization devices as in experiment 1 . On average, subjects earned 18.26 USD (including a 5 USD show-up fee). A median payoff was 20 USD. A maximum payoff was 45 USD and a minimum payoff was 5 USD.

\begin{tabular}{|c|c|c|c|c|c|c|}
\hline \multirow{2}{*}{ Pair \# } & \multicolumn{4}{|c|}{ Choice Pattern } & \multirow{2}{*}{ Conlisk $z$} & \multirow{2}{*}{$p$-value } \\
\hline & $S$ & CR & RCR & $\mathbf{R}$ & & \\
\hline 7 & 71 & 14 & 6 & 2 & 1.8106 & 0.0351 \\
\hline 8 & 58 & 9 & 23 & 3 & -2.5468 & 0.0054 \\
\hline 9 & 20 & 19 & 32 & 22 & -1.8437 & 0.0326 \\
\hline 10 & 12 & 12 & 36 & 33 & -3.6918 & $1.11 \mathrm{E}-04$ \\
\hline 11 & 3 & 5 & 31 & 54 & -4.8245 & $7.25 \mathrm{E}-07$ \\
\hline
\end{tabular}

Table 4 Observed frequency of choice patterns in experiment 2

Table 4 shows the observed frequency of four possible choice patterns in pairs 711. As expected, the frequency of the $\mathbf{S}$ pattern decreases and the frequency of the $\mathbf{R}$ pattern increases from pair 7 to pair 11 . In pair 7 , the $\mathbf{C R}$ pattern is observed more often than the $\mathbf{R C R}$ pattern. In all remaining pairs, RCR patterns outnumber $\mathbf{C R}$ patterns.

Contrary to our ex ante expectation, in pair 8, where two alternatives have exactly the same expected value, the RCR pattern is observed more often than the $\mathbf{C R}$ pattern. In both our experiments, probability information was visually presented in the form $1 / n$, with $n$ being varied. In contrast, in many previous experiments, probability information was presented as percentages or chances out of some common denominator.

\footnotetext{
${ }^{1}$ I am indebted to Ganna Pogrebna for conducting the second experiment in the US.
} 
In most cases, a visual presentation employed diagrams that work on a common denominator basis such as strips of equal length or pie charts. A different presentation format used in our experiments may be responsible for a higher incidence of RCR patterns in pair 8 , contrary to the results in the existing literature. ${ }^{2}$

For every pair of decision problems, Table 4 shows Conlisk z-statistic (Conlisk, 1989) and its $p$-value. In all five pairs there is a significant difference between RCR and CR frequencies. This difference is highly significant in pairs 10 and 11 (and comparable to $p$ values in Table 2 for corresponding pairs 2 and 3 from the first experiment).

Modal choice changes twice between a "scaled-up" and a "scaled-down" problem. In pair 9, 39 out of 93 subjects (41.9\%) opt for a sure payoff in the "scaled-up" problem but 52 out of 93 subjects (55.9\%) choose a safer lottery in the "scaled-down" problem. In pair 10 , only 24 out of 93 subjects (25.8\%) opt for a sure payoff in the "scaled-up" problem but 48 out of 93 subjects (51.6\%) choose a safer lottery in the "scaled-down" problem. Individual choice patterns ("raw" experimental data) are presented in Table 7 and Table 8 in the Appendix. 14 out of 93 subjects (15.1\%) made consistent choices in all five pairs of decision problems. Their choice patterns are presented in Table 7. Among these subjects, 7 subjects consistently choose a safer alternative in pairs 7 and 8 and a riskier alternative-in pairs 9-11. This was a modal individual choice pattern (consistent with the maximization of the expected value).

The remaining 79 subjects (84.9\%) revealed a switching choice pattern (CR or RCR) in at least one pair of decision problems. Their choice patterns are presented in Table 8. In particular, 16 subjects (17.2\%) revealed a switching behavior that may be reconciled with expected utility theory under the assumption that these subjects are exactly indifferent in one of common ratio pairs 7-11. The switching behavior of the remaining 63 subjects (67.7\%) cannot be rationalized by expected utility theory even if we assume the possibility of exact indifferences.

\footnotetext{
${ }^{2}$ This possibility has been pointed out to me by an anonymous referee whose writing style remarkably resembles that of Graham Loomes.
} 
Looking at an inconsistency rate across five "scaled-up" problems, 13 out of 93 subjects (14.0\%) made inconsistent choices. Furthermore, 22 out of 93 subjects (23.7\%) made inconsistent choices across five "scaled-down" problems. Thus, like in experiment 1, most cross-pair inconsistencies occurred in "scaled-down" problems. Overall, 31 out of 93 subjects (33.3\%) made inconsistent choices across pairs of decision problems. This inconsistency rate is higher than in experiment 1 . However, it is not unusually high. For example, Camerer (1989) reports an inconsistency rate of $31.6 \%$.

In experiment 1 we can check for consistency only across three problems. In contrast, in experiment 2 we can check for consistency across five problems. Thus, requirements for consistent choice are more stringent in experiment 2, which may explain a higher inconsistency rate in experiment 2.

Notice that an inconsistency rate across pairs (33.3\% of subjects) is still well below the switching rate within pairs ( $84.9 \% \%$ of subjects). Thus, all Results $1-3$ of the European experiment are qualitatively replicated in the second experiment in the US.

22 out of 93 subjects (23.7\%) revealed the CR pattern in at least one pair of decision problems but did not reveal the RCR pattern in any of the pairs. Another 11 subjects (11.8\%) revealed both CR and RCR patterns in different pairs of decision problems. Finally, 46 out of 93 subjects (49.5\%) revealed only RCR patterns in one or several pairs of decision problems.

Comparing the above results with the corresponding findings from experiment 1 we can conclude that the fraction of subjects who reveal both $\mathbf{C R}$ and RCR patterns is relatively low (around 11\% in both experiments). The fraction of subjects who reveal only CR pattern is notably higher in the second experiment (23.7\% vs. $10.0 \%$ in experiment 1$)$. At the same time, the fraction of subjects who reveal only RCR pattern is higher in the first experiment ( $57.1 \%$ vs. $49.5 \%$ in experiment 2 ).

A tentative explanation of these results may be the following. All subjects are of two types: those prone to the classical common ratio effect and those prone to the reverse common ratio effect. An experimentalist can reveal subjects' types by choosing an appropriate experimental design. When pairs of common ratio problems are designed so 
that a sure payoff is far above (below) the expected value of the risky lottery in the "scaled-up" problem, subjects prone to the classical (reverse) common ratio effect reveal their type in the experiment. Thus, on the aggregate level, we can observe results similar to those presented in Table 4 even though on the individual level there are relatively few subjects who simultaneously exhibit both types of the common ratio effect.

\section{Conclusion}

This paper presents three results from a new experimental study on choice under risk. First, there are certain decision problems where people choose a lottery over a sure payoff but switch to choosing a safer alternative when the probabilities of all non-zero outcomes are scaled down by the same common ratio. Second, such a reverse common ratio effect is observed when the same lottery is compared vis-à-vis several different sure monetary payoffs. Third, switching rates within common ratio problems are much higher compared to the rates of inconsistency across these decision problems.

The first result establishes the existence of a systematic reverse common ratio effect. The second result effectively states that this effect is not a mere artifact of exact indifferences. Finally, the third result demonstrates that the reverse common ratio effect cannot be attributed to random errors or noise. All three results appear to be quite novel and undocumented in the existing literature.

Expected utility theory cannot account for Result 1 in conjunction with Result 2. In this regard, most non-expected utility theories do not offer any comparative advantage over expected utility theory. Only a perceived relative argument model (Loomes, 2008) is partially successful in explaining new experimental data.

Models of probabilistic choice face a different challenge. They fail to account for Result 1 in conjunction with Result 3. For example, a strong utility model and stochastic expected utility theory (Blavatskyy, 2007) can rationalize Result 1 but they also predict too many inconsistencies across pairs of common ratio problems. A model of imprecise preferences (MacCrimmon and Smith, 1986) appears to be the most promising alternative-it is consistent with Results 1 and 3 but does not explicitly predict Result 1 . 
The results of a control experiment show that subjects can exhibit both the classical and the reverse common ratio effect at the same time, at least on the aggregate level. Many people choose a sure payoff over a risky lottery if the expected value of the lottery is far below the sure payoff. However, quite a few people also switch to choosing a riskier alternative when the probabilities of all non-zero outcomes are scaled down by the same common ratio. Very few people reveal the reverse common ratio effect in this case. These findings are in line with the results from the existing experiments.

At the same time, many people choose a risky lottery over a sure payoff if the expected value of the lottery is far above the sure payoff. However, many of them also switch to choosing a safer alternative when the probabilities of all non-zero outcomes are scaled down by the same common ratio. A significantly smaller fraction of people reveal the standard common ratio effect in this case.

The novel results of this paper have potentially far reaching implications. First, we need more experimental research with "unusual" common ratio problems of the type studied in this paper. Second, we need more theoretical work, which is not narrowly focused on the standard Allais examples. Hopefully along these lines, a general and descriptively adequate theory of decision making under risk will emerge one day.

\section{References}

Allais, Maurice (1953) “Le Comportement de l'Homme Rationnel devant le Risque: Critique des Postulates et Axiomes de l’Ecole Américaine" Econometrica 21, 503-546 Ballinger, Parker and Nathaniel Wilcox (1997) "Decisions, error and heterogeneity" Economic Journal 107, 1090-1105

Battalio Raymond, John Kagel and Komain Jiranyakul (1990) "Testing between Alternative Models of Choice under Uncertainty: Some Initial Results" Journal of Risk and Uncertainty 3, 25-50

Blavatskyy, Pavlo (2007) "Stochastic Expected Utility Theory" Journal of Risk and Uncertainty 34, 259-286

Butler, David and Graham Loomes (2007) "Imprecision as an Account of the Preference Reversal Phenomenon" American Economic Review 97(1), 277-297 
Camerer, Colin (1989) "An experimental test of several generalized utility theories." Journal of Risk and Uncertainty 2, 61-104

Conlisk, John (1989) "Three Variants on the Allais Example" American Economic Review 79 (3), 392-407

Gul, Faruk (1991) “A Theory of Disappointment Aversion” Econometrica 59, 667-686

Gul, Faruk and Wolfgang Pesendorfer (2006) "Random Expected Utility" Econometrica 71, $121-146$

Kahneman, Daniel and Amos Tversky (1979) "Prospect Theory: an Analysis of Decision under Risk" Econometrica 47, 263-291

Loomes, Graham (2008) “Modelling Choice and Valuation in Decision Experiments", University of East Anglia working paper

Loomes, Graham (2005) “Modelling the Stochastic Component of Behavior in Experiments: Some Issues for the Interpretation of Data" Experimental Economics 8, 301-323

Loomes, Graham and Robert Sugden (1998) "Testing Different Stochastic Specifications of Risky Choice" Economica 65, 581-598

Loomes, Graham and Robert Sugden (1995) "Incorporating a stochastic element into decision theories" European Economic Review 39, 641-648

MacCrimmon, Kenneth and Maxwell Smith (1986) "Imprecise Equivalences: Preference Reversals in Money and Probability", University of British Columbia working paper \#1211

Quiggin, John (1981) "Risk perception and risk aversion among Australian farmers" Australian Journal of Agricultural Recourse Economics 25, 160-169

Tversky, Amos and Daniel Kahneman (1992) “Advances in Prospect Theory: Cumulative Representation of Uncertainty" Journal of Risk and Uncertainty 5, 297-323

Viscusi, Kip (1989) "Prospective Reference Theory: Toward an Explanation of the Paradoxes" Journal of Risk and Uncertainty 2, 235-264

Yaari, Menahem (1987) “The Dual Theory of Choice under Risk" Econometrica 55, 95-115 


\section{Appendix}

\section{Instructions}

Welcome to our experiment! This is an experiment in decision theory. This experiment is financed from research funds. In this experiment we would like to ask you to take a number of decisions.

At the end of the experiment we will determine how much money you get. Your payoff depends only on your decisions and the realization of random events. Your payoff does not depend on the decisions of other participants. Your anonymity will be preserved during and after the experiment.

During the experiment you need to answer a number of questions that appear on your computer screen one by one. Please note that that there are no right or wrong answers in this experiment. Here is an example of a typical question that you may receive during the experiment:

\section{Question 1}

Please choose your preferred alternative:

\begin{tabular}{|l|l|}
\hline $\begin{array}{l}\text { Left Alternative } \\
\text { An experimenter shows you } 4 \text { cards. }\end{array}$ & $\begin{array}{l}\text { Right Alternative } \\
\text { One card is red and } 3 \text { cards are black. } \\
\text { All } 4 \text { cards are faced down and reshuffled. } \\
\text { Then you draw one card. You receive } \$ 80 \text { if it is } \\
\text { red and you receive nothing if it is black. }\end{array}$ \\
\hline
\end{tabular}

\section{I choose: $\quad \square$ Left Alternative}

\section{Right Alternative}

At the end of the experiment you have to spin a roulette wheel to randomly select one question number. This question will be used to determine your payoff. For example, suppose that the roulette wheel stops at number 1 . Then question 1 is selected. Suppose that your question 1 is a question shown above. If you have chosen the right alternative in this question, you receive $\$ 20$. If you have chosen the left alternative in this question, you need to draw one of four cards. You receive $\$ 80$ if your drawn card is red and you receive nothing if your drawn card is black. Please note that any question can be randomly selected at the end to determine your payoff. So it is in your best interest to answer all questions carefully. 


\begin{tabular}{|c|c|c|c|c|c|c|c|c|c|c|c|c|}
\hline \multirow{2}{*}{ Subject ID } & \multicolumn{2}{|c|}{ Pair 1} & \multicolumn{2}{|c|}{ Pair 2} & \multicolumn{2}{|c|}{ Pair 3} & \multicolumn{2}{|c|}{ Pair 4} & \multicolumn{2}{|c|}{ Pair 5} & \multicolumn{2}{|c|}{ Pair 6} \\
\hline & up & down & up & down & up & down & up & down & up & down & up & down \\
\hline \#8, \#50, \#55 & 1 & 1 & 1 & 1 & 0 & 1 & 1 & 1 & 1 & 1 & 0 & 1 \\
\hline \#1, \#43 & 0 & 0 & 0 & 0 & 0 & 0 & 1 & 1 & 0 & 1 & 0 & 1 \\
\hline \#2, \#22 & 0 & 1 & 0 & 0 & 0 & 0 & 1 & 1 & 1 & 1 & 0 & 0 \\
\hline \#4, \#61 & 0 & 1 & 0 & 1 & 0 & 1 & 1 & 1 & 1 & 1 & 1 & 1 \\
\hline$\# 5, \# 52$ & 0 & 1 & 0 & 1 & 0 & 1 & 1 & 1 & 1 & 1 & 1 & 0 \\
\hline \#9, \#19 & 0 & 1 & 0 & 1 & 0 & 0 & 1 & 1 & 1 & 1 & 0 & 1 \\
\hline \#10, \#56 & 0 & 1 & 0 & 1 & 0 & 1 & 1 & 1 & 1 & 1 & 0 & 0 \\
\hline \#11, \#60 & 1 & 1 & 0 & 1 & 0 & 1 & 1 & 1 & 1 & 1 & 1 & 1 \\
\hline \#18, \#66 & 0 & 1 & 0 & 0 & 0 & 1 & 1 & 1 & 1 & 1 & 1 & 1 \\
\hline \#20, \#39 & 0 & 0 & 0 & 0 & 0 & 0 & 1 & 1 & 0 & 1 & 0 & 0 \\
\hline \#31, \#57 & 0 & 1 & 0 & 1 & 0 & 0 & 1 & 1 & 1 & 1 & 0 & 0 \\
\hline \#38, \#62 & 1 & 1 & 1 & 0 & 1 & 0 & 1 & 1 & 1 & 1 & 0 & 0 \\
\hline \#7 & 0 & 1 & 0 & 1 & 0 & 1 & 1 & 1 & 1 & 1 & 0 & 1 \\
\hline \#12 & 0 & 1 & 1 & 1 & 0 & 1 & 1 & 1 & 1 & 1 & 0 & 0 \\
\hline \#13 & 1 & 1 & 1 & 1 & 0 & 1 & 1 & 1 & 1 & 1 & 1 & 1 \\
\hline \#21 & 1 & 1 & 1 & 1 & 1 & 1 & 1 & 1 & 1 & 1 & 0 & 1 \\
\hline$\# 23$ & 1 & 1 & 0 & 0 & 0 & 1 & 1 & 1 & 1 & 1 & 1 & 1 \\
\hline \#24 & 0 & 1 & 0 & 0 & 0 & 1 & 1 & 1 & 1 & 1 & 1 & 0 \\
\hline$\# 27$ & 0 & 0 & 1 & 1 & 0 & 1 & 1 & 1 & 1 & 1 & 1 & 1 \\
\hline$\# 29$ & 0 & 0 & 0 & 1 & 0 & 1 & 1 & 1 & 1 & 1 & 0 & 0 \\
\hline \#30 & 0 & 0 & 0 & 0 & 0 & 0 & 1 & 0 & 0 & 0 & 0 & 0 \\
\hline \#32 & 1 & 0 & 0 & 0 & 0 & 0 & 1 & 1 & 0 & 1 & 0 & 0 \\
\hline \#34 & 0 & 0 & 0 & 0 & 0 & 0 & 1 & 1 & 1 & 1 & 0 & 1 \\
\hline \#35 & 0 & 0 & 0 & 0 & 0 & 0 & 0 & 1 & 0 & 0 & 0 & 1 \\
\hline \#36 & 0 & 1 & 0 & 0 & 0 & 0 & 1 & 1 & 1 & 1 & 0 & 1 \\
\hline \#37 & 0 & 1 & 0 & 1 & 0 & 0 & 1 & 1 & 0 & 1 & 0 & 1 \\
\hline$\# 40$ & 0 & 0 & 0 & 0 & 0 & 0 & 1 & 1 & 1 & 0 & 1 & 0 \\
\hline \#41 & 0 & 0 & 0 & 1 & 0 & 0 & 1 & 1 & 1 & 1 & 0 & 1 \\
\hline \#42 & 1 & 1 & 1 & 1 & 1 & 0 & 1 & 1 & 1 & 1 & 1 & 1 \\
\hline \#44 & 0 & 1 & 0 & 1 & 0 & 0 & 1 & 1 & 0 & 1 & 1 & 1 \\
\hline \#46 & 0 & 0 & 0 & 1 & 0 & 0 & 1 & 1 & 1 & 1 & 0 & 0 \\
\hline \#48 & 1 & 1 & 1 & 0 & 0 & 0 & 1 & 1 & 0 & 1 & 0 & 0 \\
\hline \#49 & 0 & 0 & 0 & 0 & 0 & 0 & 0 & 1 & 0 & 1 & 0 & 0 \\
\hline \#51 & 1 & 1 & 0 & 1 & 0 & 1 & 1 & 1 & 1 & 1 & 0 & 1 \\
\hline$\# 54$ & 0 & 0 & 0 & 0 & 1 & 0 & 1 & 1 & 0 & 1 & 0 & 0 \\
\hline \#58 & 1 & 1 & 0 & 1 & 0 & 1 & 1 & 1 & 1 & 1 & 1 & 0 \\
\hline \#63 & 1 & 1 & 1 & 0 & 0 & 0 & 1 & 1 & 1 & 1 & 0 & 0 \\
\hline \#65 & 0 & 1 & 0 & 0 & 0 & 1 & 1 & 1 & 1 & 1 & 0 & 0 \\
\hline \#67 & 1 & 0 & 0 & 0 & 0 & 0 & 1 & 1 & 0 & 0 & 0 & 0 \\
\hline \#68 & 0 & 1 & 0 & 0 & 1 & 1 & 1 & 1 & 1 & 0 & 0 & 1 \\
\hline \#69 & 0 & 1 & 0 & 0 & 0 & 1 & 0 & 1 & 0 & 1 & 0 & 1 \\
\hline$\# 70$ & 1 & 1 & 0 & 1 & 0 & 0 & 1 & 1 & 0 & 1 & 0 & 1 \\
\hline
\end{tabular}

Table 5 Individual choice patterns with switching in Experiment 1

( 1 denotes choice of a safer lottery) 


\begin{tabular}{|c|c|c|c|c|c|c|c|c|c|c|c|c|}
\hline \multirow{2}{*}{ Subject ID } & \multicolumn{2}{|c|}{ Pair 1} & \multicolumn{2}{|c|}{ Pair 2} & \multicolumn{2}{|c|}{ Pair 3} & \multicolumn{2}{|c|}{ Pair 4} & \multicolumn{2}{|c|}{ Pair 5} & \multicolumn{2}{|c|}{ Pair 6} \\
\hline & up & down & up & down & up & down & up & down & up & down & up & down \\
\hline $\begin{array}{l}\# 17, \# 26, \# 45, \\
\# 47, \# 64\end{array}$ & 1 & 1 & 1 & 1 & 1 & 1 & 1 & 1 & 1 & 1 & 1 & 1 \\
\hline \#15, \#28, \#59 & 0 & 0 & 0 & 0 & 0 & 0 & 1 & 1 & 0 & 0 & 0 & 0 \\
\hline$\# 3, \# 25$ & 0 & 0 & 0 & 0 & 0 & 0 & 1 & 1 & 1 & 1 & 0 & 0 \\
\hline \#6 & 1 & 1 & 1 & 1 & 0 & 0 & 1 & 1 & 1 & 1 & 0 & 0 \\
\hline \#14 & 0 & 0 & 0 & 0 & 0 & 0 & 1 & 1 & 1 & 1 & 1 & 1 \\
\hline \#16 & 1 & 1 & 1 & 1 & 1 & 1 & 1 & 1 & 1 & 1 & 0 & 0 \\
\hline \#33 & 0 & 0 & 0 & 0 & 0 & 0 & 0 & 0 & 0 & 0 & 0 & 0 \\
\hline \#53 & 1 & 1 & 0 & 0 & 0 & 0 & 1 & 1 & 1 & 1 & 1 & 1 \\
\hline
\end{tabular}

Table 6 Individual choice patterns without switching in Experiment 1

(1 denotes choice of a safer lottery)

\begin{tabular}{|l|cc|cc|cc|cc|cc|}
\hline \multirow{2}{*}{ Subject ID } & \multicolumn{2}{|c|}{ Pair 7 } & \multicolumn{2}{c|}{ Pair 8 } & \multicolumn{2}{c|}{ Pair 9 } & \multicolumn{2}{c|}{ Pair 10 } & \multicolumn{2}{c|}{ Pair 11 } \\
\cline { 2 - 10 } & up & down & up down & up down & up & down & up down \\
\hline$\# 31, \# 43, \# 56, \# 71$, & & & & & & & & & & \\
$\# 81, \# 89, \# 17$ & 1 & 1 & 1 & 1 & 0 & 0 & 0 & 0 & 0 & 0 \\
$\# 16, \# 24, \# 51$ & 1 & 1 & 1 & 1 & 1 & 1 & 1 & 1 & 0 & 0 \\
$\# 54, \# 64$ & 1 & 1 & 0 & 0 & 0 & 0 & 0 & 0 & 0 & 0 \\
$\# 85$ & 1 & 1 & 1 & 1 & 1 & 1 & 1 & 1 & 1 & 1 \\
$\# 6$ & 1 & 1 & 1 & 1 & 1 & 1 & 0 & 0 & 0 & 0 \\
\hline
\end{tabular}

Table 7 Individual choice patterns without switching in Experiment 2

(1 denotes choice of a safer lottery) 


\begin{tabular}{|c|c|c|c|c|c|c|c|c|c|c|}
\hline \multirow{2}{*}{ Subject ID } & \multicolumn{2}{|c|}{ Pair 7} & \multicolumn{2}{|c|}{ Pair 8} & \multicolumn{2}{|c|}{ Pair 9} & \multicolumn{2}{|c|}{ Pair 10} & \multicolumn{2}{|c|}{ Pair 11} \\
\hline & up & down & up & down & up & down & up & down & up & down \\
\hline$\# 18, \# 36, \# 42, \# 68, \# 87, \# 92$ & 1 & 1 & 1 & 1 & 1 & 1 & 0 & 1 & 0 & 1 \\
\hline \#5, \#39, \#61, \#75, \#84 & 1 & 1 & 0 & 1 & 0 & 1 & 0 & 1 & 0 & 1 \\
\hline$\# 21, \# 26, \# 55, \# 72$ & 1 & 1 & 0 & 1 & 0 & 1 & 0 & 0 & 0 & 0 \\
\hline$\# 28, \# 38, \# 58, \# 93$ & 1 & 1 & 0 & 1 & 0 & 1 & 0 & 1 & 0 & 0 \\
\hline$\# 12, \# 37, \# 52, \# 65$ & 1 & 1 & 1 & 1 & 0 & 1 & 0 & 1 & 0 & 1 \\
\hline \#1, \#53, \#91 & 1 & 1 & 1 & 1 & 1 & 1 & 0 & 1 & 0 & 0 \\
\hline$\# 25$, \#49, \#60 & 1 & 1 & 1 & 1 & 1 & 0 & 0 & 0 & 0 & 0 \\
\hline$\# 11, \# 35, \# 63$ & 0 & 1 & 0 & 1 & 0 & 1 & 0 & 1 & 0 & 1 \\
\hline$\# 13, \# 66$ & 1 & 1 & 1 & 1 & 1 & 1 & 1 & 1 & 0 & 1 \\
\hline \#3, \#59 & 1 & 0 & 1 & 1 & 1 & 0 & 1 & 0 & 0 & 0 \\
\hline \#33, \#67 & 1 & 1 & 1 & 1 & 0 & 0 & 1 & 0 & 0 & 0 \\
\hline \#34, \#47 & 1 & 1 & 1 & 1 & 0 & 1 & 0 & 0 & 0 & 0 \\
\hline$\# 40, \# 83$ & 1 & 1 & 1 & 1 & 0 & 1 & 0 & 1 & 0 & 0 \\
\hline$\# 45, \# 70$ & 1 & 1 & 0 & 1 & 0 & 0 & 0 & 0 & 0 & 0 \\
\hline \#46, \#62 & 1 & 1 & 1 & 0 & 0 & 0 & 0 & 0 & 0 & 0 \\
\hline \#77, \#79 & 1 & 1 & 1 & 1 & 1 & 0 & 0 & 0 & 1 & 0 \\
\hline$\# 10$ & 1 & 0 & 0 & 0 & 0 & 1 & 0 & 0 & 0 & 0 \\
\hline \#14 & 1 & 1 & 1 & 1 & 1 & 1 & 1 & 0 & 0 & 0 \\
\hline \#15 & 1 & 0 & 1 & 1 & 1 & 1 & 1 & 1 & 0 & 0 \\
\hline \#19 & 1 & 1 & 1 & 1 & 1 & 1 & 0 & 0 & 0 & 1 \\
\hline$\# 2$ & 1 & 0 & 1 & 1 & 1 & 0 & 1 & 0 & 1 & 1 \\
\hline \#20 & 1 & 1 & 1 & 0 & 0 & 1 & 0 & 0 & 0 & 0 \\
\hline \#22 & 1 & 1 & 1 & 1 & 1 & 1 & 1 & 1 & 1 & 0 \\
\hline \#23 & 1 & 0 & 1 & 0 & 1 & 0 & 0 & 0 & 0 & 1 \\
\hline$\# 27$ & 1 & 1 & 1 & 1 & 0 & 0 & 0 & 0 & 0 & 1 \\
\hline$\# 29$ & 1 & 1 & 1 & 1 & 1 & 0 & 1 & 1 & 0 & 0 \\
\hline$\# 30$ & 1 & 1 & 1 & 0 & 1 & 0 & 1 & 0 & 0 & 0 \\
\hline$\# 32$ & 0 & 1 & 0 & 1 & 0 & 1 & 0 & 1 & 0 & 0 \\
\hline$\# 4$ & 1 & 0 & 1 & 1 & 1 & 0 & 1 & 0 & 0 & 1 \\
\hline \#41 & 0 & 1 & 0 & 1 & 0 & 1 & 1 & 1 & 0 & 1 \\
\hline \#44 & 1 & 1 & 1 & 1 & 1 & 0 & 0 & 1 & 0 & 1 \\
\hline \#48 & 1 & 1 & 1 & 0 & 0 & 0 & 0 & 1 & 0 & 0 \\
\hline \#50 & 1 & 1 & 1 & 1 & 1 & 0 & 1 & 0 & 0 & 0 \\
\hline \#57 & 1 & 0 & 1 & 1 & 1 & 0 & 0 & 1 & 1 & 1 \\
\hline$\# 69$ & 0 & 0 & 1 & 0 & 0 & 1 & 0 & 0 & 1 & 0 \\
\hline \#7 & 0 & 0 & 1 & 0 & 1 & 0 & 0 & 0 & 1 & 0 \\
\hline$\# 73$ & 1 & 1 & 1 & 1 & 0 & 0 & 0 & 1 & 0 & 1 \\
\hline \#74 & 1 & 0 & 1 & 1 & 0 & 0 & 1 & 0 & 0 & 1 \\
\hline \#76 & 1 & 1 & 1 & 1 & 0 & 1 & 1 & 1 & 0 & 0 \\
\hline \#78 & 1 & 0 & 0 & 1 & 0 & 0 & 0 & 1 & 0 & 0 \\
\hline$\# 8$ & 1 & 1 & 0 & 1 & 0 & 0 & 0 & 1 & 0 & 1 \\
\hline$\# 80$ & 1 & 1 & 0 & 1 & 0 & 1 & 1 & 1 & 0 & 0 \\
\hline \#82 & 1 & 0 & 1 & 1 & 1 & 0 & 0 & 0 & 0 & 0 \\
\hline$\# 86$ & 0 & 1 & 1 & 1 & 0 & 1 & 0 & 1 & 0 & 1 \\
\hline \#88 & 1 & 0 & 1 & 0 & 1 & 0 & 1 & 0 & 0 & 0 \\
\hline$\# 9$ & 1 & 0 & 1 & 1 & 0 & 0 & 1 & 0 & 0 & 0 \\
\hline \#90 & 1 & 0 & 1 & 1 & 1 & 0 & 0 & 1 & 0 & 1 \\
\hline
\end{tabular}

Table 8 Individual choice patterns with switching in Experiment 2 ( 1 denotes choice of a safer lottery) 